\title{
Results of a venous thromboembolism prophylaxis program for hospitalized patients
}

\author{
This article was published in the following Dove Press journal: \\ Vascular Health and Risk Management \\ 12 December 2016 \\ Number of times this article has been viewed
}

\author{
Luiz Francisco Cardoso \\ Daniella Vianna C Krokoscz \\ Edison Ferreira de Paiva \\ Ilka Spinola Furtado \\ Jorge Mattar Jr \\ Marcia Martiniano de \\ Souza e Sá \\ Antonio Carlos Onofre \\ de Lira \\ Sírio Libanês Hospital, São Paulo, \\ Brazil
}

Correspondence: Luiz Francisco Cardoso Sírio Libanês Hospital, Rua Dona Adma Jafet, 9I, Bela Vista, São Paulo CEP 0I 308-050, Brazil

Tel +55 II 33945886

Email luiz.cardoso@hsl.org.br
Introduction: Venous thromboembolism (VTE) is the leading cause of preventable death in hospitalized patients. However, existing prophylaxis guidelines are rarely followed.

Objective: The aim of the study was to present and discuss implementation strategies and the results of a VTE prophylaxis program for medical and surgical patients admitted to a large general hospital.

Patients and methods: This prospective observational study was conducted to describe the strategy used to implement a VTE prophylaxis program in hospitalized medical and surgical patients and to analyze the results in terms of the risk assessment rate within the first 24 hours after admission, adequacy of the prophylaxis prescription, and prevalence of VTE in the discharge records before and after program implementation. We used the Mantel-Haenszel chi-square test for the linear trend of the data analysis and set the significance level to $P<0.05$.

Results: With the support of an institutional VTE prophylaxis committee, a multiple-strategy approach was used in the implementation of the protocol, which included continuing education, complete data recording using computerized systems, and continuous auditing of and feedback to the medical staff and multidisciplinary teams. Approximately $90 \%$ of patients were evaluated within the first 24 hours after admission, and no significant difference in this percentage was observed among the years analyzed. A progressive increase in adherence to protocol recommendations, from $63.8 \%$ in 2010 to $75.0 \%$ in $2014(P<0.001)$, was noted. The prevalence of symptomatic VTE in the discharge records of patients decreased from $2.03 \%$ in 2009 to $1.69 \%$ in $2014(P=0.033)$

Conclusion: The implementation of a VTE prophylaxis program targeting adult patients admitted to a large hospital employing a multiple-strategy approach achieved high rates of risk assessment within 24 hours of admission, improved the adherence to prophylaxis recommendations in high-risk patients, and reduced the rate of VTE events in the discharge records. Keywords: deep venous thrombosis, pulmonary embolism, prevention and control, patient safety, quality control

\section{Introduction}

The term venous thromboembolism (VTE) encompasses both deep-vein thrombosis and its most severe complication, pulmonary embolism (PE). Although these are potentially avoidable events, ${ }^{1}$ it is believed that PEs cause up to $10 \%$ of in-hospital deaths ${ }^{2}$ and that $\sim 25 \%$ of all VTE cases are associated with hospital admission, accounting for $75 \%$ of medical patients. ${ }^{3}$ Post-thrombotic syndrome and pulmonary hypertension are frequent chronic complications and cause much discomfort to patients, in addition to imposing high costs to the health care system. ${ }^{4}$ 
Although available for $>20$ years, the recommendations for VTE prophylaxis in medical and surgical patients are rarely applied. ${ }^{5}$ One recent study conducted in 358 hospitals from 32 countries involving $\sim 70,000$ patients showed that $\sim 50 \%$ of hospitalized patients were at risk for VTE, but only half received prophylaxis. ${ }^{6}$ The reasons ranged from ignorance of the guidelines to socioeconomic problems and included resistance to changes to long-established practices, disagreement with the recommendations, and fear of causing hemorrhagic complications. ${ }^{7}$ The lack of institutional policies that encourage the application of prophylaxis contributes to the continuation of the current scenario. ${ }^{7}$ In addition, changing this situation is not easy, and widely encompassing, multiple-strategy approaches are recommended, including continued education, distribution of educational materials, the use of risk assessment instruments that are simple to apply, and continuous reevaluation of outcomes.

The objective of the present study was to present and discuss implementation strategies and the results of a VTE prophylaxis program for medical and surgical patients admitted to a large general hospital.

\section{Patients and methods}

The study was conducted at the Syrian-Lebanese Hospital (Hospital Sírio Libanês, HSL), São Paulo, Brazil, from March 2010 to March 2014. The HSL is a philanthropic open-staff hospital with 450 beds for intensive care, clinical, surgical, and pediatric ward services. It includes a VTE prophylaxis committee composed of physicians, nurses, physical therapists, and pharmacists. An institutional protocol for VTE prophylaxis for hospitalized medical and surgical patients was instituted in August 2009. The study was approved by the Syrian-Lebanese Hospital Ethics and Research Committee (No 1029.341) on April 16, 2015 and an individual informed consent was not required.

During the implementation period in 2010, the following strategies were applied to improve the adherence to the protocol recommendations: 1) scientific meetings, 2) practical training on computerized tools, and 3) admissional and permanent training program for physicians, nurses, and physiotherapists. The main aspects highlighted were the risk factors of thrombosis and chemical and mechanical strategies of prevention and complications. In addition, practical training was conducted by the information technology department staff.

Beginning in March 2010, the data related to VTE prophylaxis and the gradual implementation of the protocol in the various hospital sections were recorded in a computerized database. The protocol was adapted to the hospital's electronic medical records system, and the doctors and nurses contracted by the hospital were trained in the use of the system and charged with assessing the VTE risk in all patients. Pharmacists, physical therapists, and open-staff physicians participated in continued education activities. This process continued until December 2010, and for the purposes of the current study, this period is called the implementation period. Once the training period ended, the post-implementation period began, lasting from January 2011 to March 2014. The data related to pharmacological and/or mechanical prophylaxis collected from the electronic medical records were used to determine whether the prophylaxis recommendations based on the risk assessment were followed. In addition, the prevalence of VTE as a primary diagnosis at the time of discharge of all hospitalized patients in 2009 , ie, before the implementation of the protocol, and throughout the study period was recorded.

\section{Risk assessment}

Considering surgical patients, the risk of VTE depends on the age and the surgery performed, in association with the existing risk factors. For clinical patients, the prophylaxis is indicated to $\geq 40$-year-old patients with mobility reduction and at least one risk factor. Patients younger than 40 years old should have assessed the risk individually. The recommendations adopted at Syrian-Lebanese Hospital followed the $8^{\text {th }}$ Consensus of American College of Chest Physicians Guidelines $\left(8^{\text {th }} \mathrm{ACCP}\right){ }^{3}$ The protocol was not modified during the period of this study.

Assessments were performed within the first 24 hours after admission for all hospitalized patients, except for those under palliative care, already using anticoagulants, or presenting VTE. The assessments were performed by the oncall attending physicians in the intensive and semi-intensive care units and by nurses in the wards. The assessment was repeated whenever a patient exhibited changes in their functional status or was transferred to another hospital section. Following each assessment, the system generated a report with recommendations for prophylaxis, which was printed and filed together with the patient's medical records, to orient the prescribing doctor.

\section{Adherence to the protocol}

Twenty-four hours after the risk assessment, the data regarding the recommended and effectively prescribed prophylaxis were cross-checked. From March 2010 to August 2012, the adherence to the protocol was analyzed based on the verification of a prescription of chemical and/or mechanical prophylaxis at 
any time during the hospital stay. Starting in September 2012, the analysis of adherence was changed, and the prescription of chemical and/or mechanical prophylaxis was investigated on a daily basis. The adherence to the prophylaxis protocol was assessed considering the risk of each individual patient. For patients considered high risk of thrombosis, the use of chemical prophylaxis was verified. If the patient was considered as low risk and had no prescription of chemical prophylaxis, it was also considered in according to the protocol recommendation. The VTE prophylaxis committee met monthly to evaluate the results and discuss strategies for change.

\section{Prevalence of VTE events}

The prevalence of VTE was calculated for all study time points (years) using the hospital information system and was based on the discharge diagnosis according to the tenth revision of the International Statistical Classification of Diseases and Related Health Problems (IDC-10) formulated by the World Health Organization. ${ }^{8}$

\section{Statistical analysis}

Data stored in the hospital information system were extracted and analyzed using Business Intelligence- QlikView $^{\circledR}$ (Qlik Technologies Inc., Philadelphia, PA, USA, 1993) software. The index of performance of VTE risk assessments was calculated for all study time points according to the following equation:

Number of adult patients subjected to VTE risk assessment within the first 24 hours after admission per year

Number of hospitalized adult patients

The index of adherence to the pharmacological or mechanical prophylaxis method recommended by the protocol was calculated for all study time points according to the following equation:

Number of patients - days the recommended prophylaxis was prescribed per year

Number of patients - days of assessed $\times 100$ adult patients per year

The prevalence of VTE was calculated for all study time points according to the following equation:

$$
\begin{aligned}
& \text { Number of patients discharged } \\
& \text { with VTE diagnosis } \\
& \text { Total number of discharged } \\
& \text { patients per year }
\end{aligned}
$$

Table I Demographic and clinical characteristics of patients assessed for VTE risk from 2010 to 2014

\begin{tabular}{ll}
\hline Sex & \\
Male & $52.2 \%$ \\
Female & $47.8 \%$ \\
Total & $100.0 \%$ \\
Age (years) & \\
$<40$ & $18.1 \%$ \\
$\geq 40$ and $<60$ & $32.4 \%$ \\
$\geq 60$ and $<75$ & $29.1 \%$ \\
$\geq 75$ & $20.4 \%$ \\
Total & $100.0 \%$ \\
Diagnostic group & \\
Neoplasms & $28.44 \%$ \\
Digestive & $13.28 \%$ \\
Circulatory & $13.28 \%$ \\
Genitourinary & $10.68 \%$ \\
Musculoskeletal & $7.09 \%$ \\
Respiratory & $6.14 \%$ \\
External causes & $6.12 \%$ \\
Nervous system & $2.59 \%$ \\
Infectious & $2.37 \%$ \\
Others & $9.92 \%$ \\
Total & $100.0 \%$ \\
Department & \\
Clinical & $48.5 \%$ \\
Surgical & $51.5 \%$ \\
Total & $100.0 \%$ \\
\hline Abreviation: $V T E, ~$ &
\end{tabular}

Abbreviation: VTE, venous thromboembolism.

Comparisons were performed using the Mantel-Haenszel chi-square test for linear trend, and the significance level was set to $P<0.05$.

\section{Results}

All clinical or surgical adult patients admitted from March 2010 to March 2014 were analyzed. Demographic and clinical characteristics of patients assessed for VTE risk from 2010 to 2014 are shown in Table 1. The absolute number of patients subjected to VTE risk assessment within the first 24 hours after admission and the total number of hospitalized patients are shown in Figure 1. Figure 2 shows the percentage of patients assessed for VTE risk per year. Approximately $90 \%$ of patients were assessed throughout the study period, and no significant difference was observed among the analyzed years.

The percentage of adherence to the protocol per year is shown in Figure 3. The percentage of adherence to the protocol recommendations increased progressively over the study period and became statistically significant in $2013(P<0.001$ compared to the period before 2013).

The prevalence rates of VTE per number of discharged patients before the implementation of the protocol in 2009 


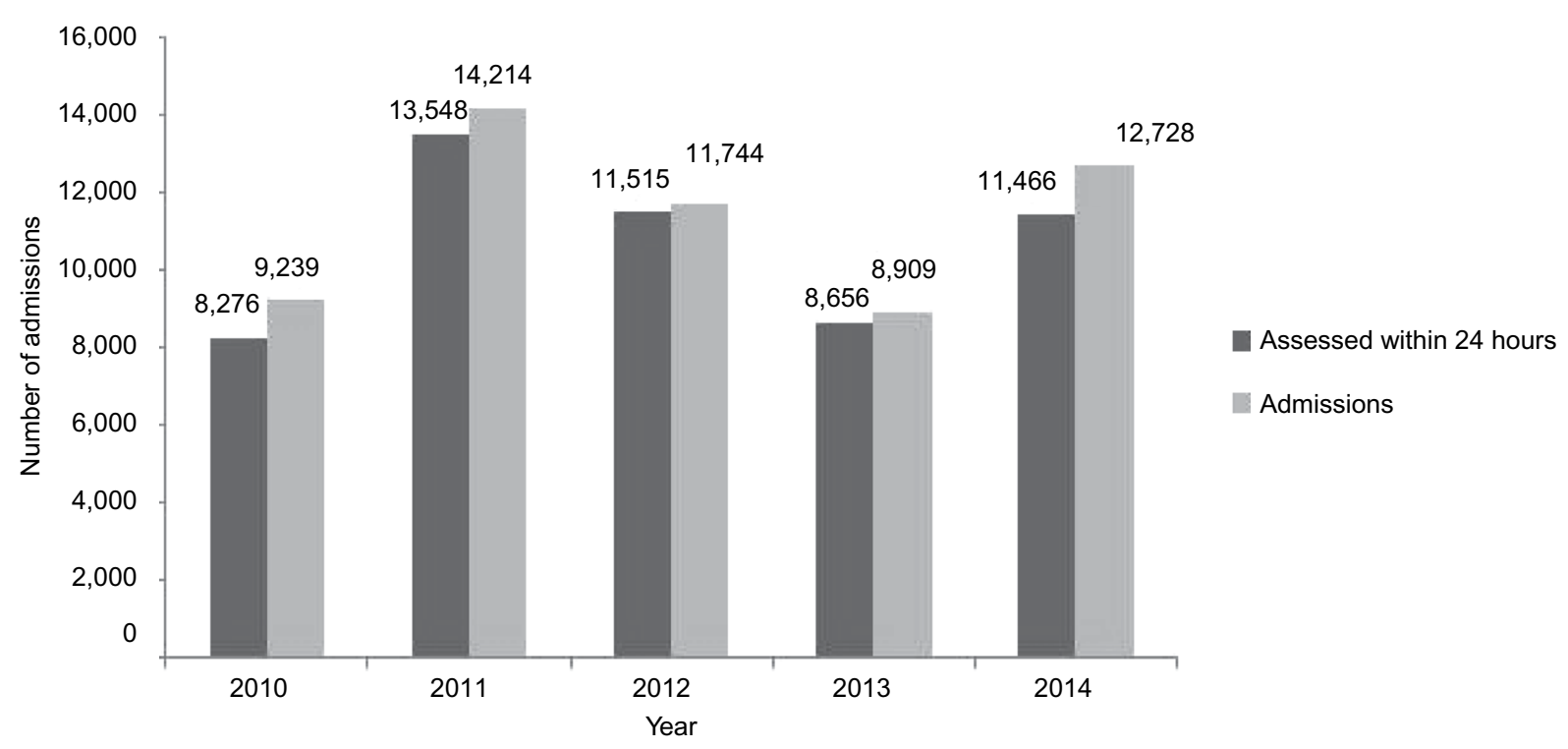

Figure I Absolute number of patients assessed for VTE risk within 24 hours after admission and total number of admitted adult patients from 20 I0 to 20 I4. Abbreviation: VTE, venous thromboembolism.

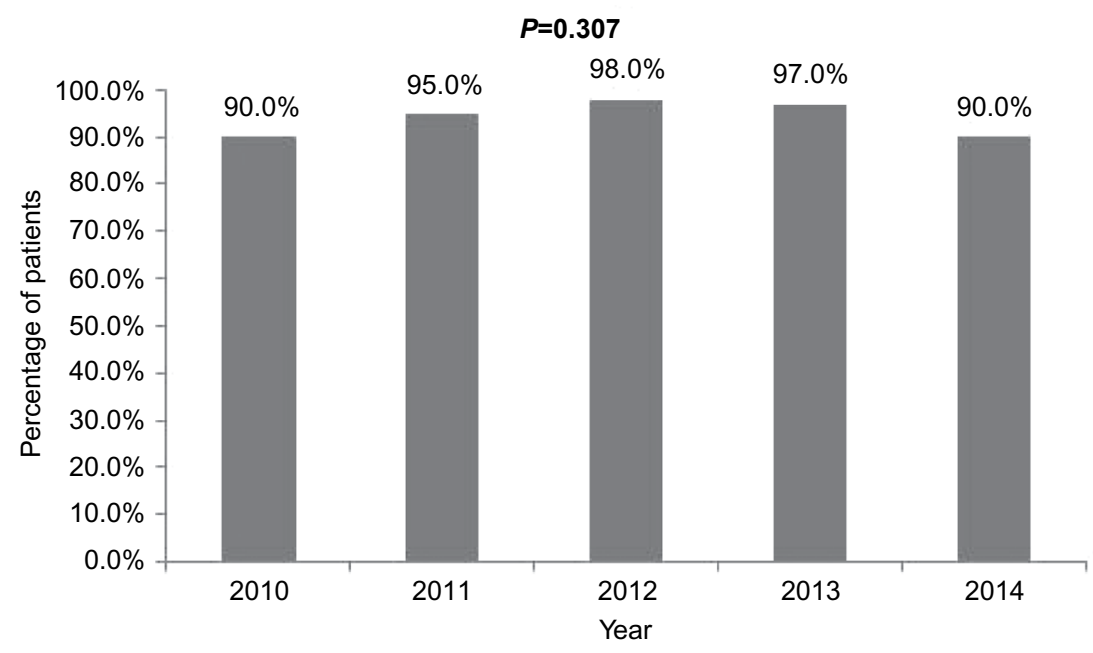

Figure 2 Percent of patients assessed for VTE risk within 24 hours after admissions from 2010 to 2014. Abbreviation: VTE, venous thromboembolism.

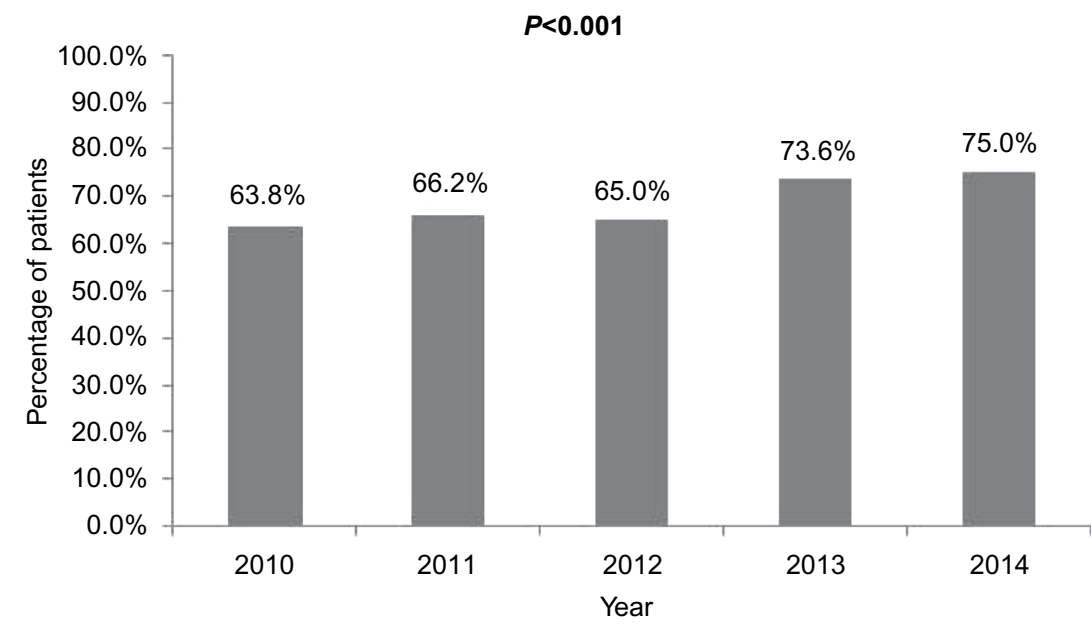

Figure 3 Percent of patients adherence to chemical and/or mechanical prophylaxis recommendations from 2010 to 2014. 


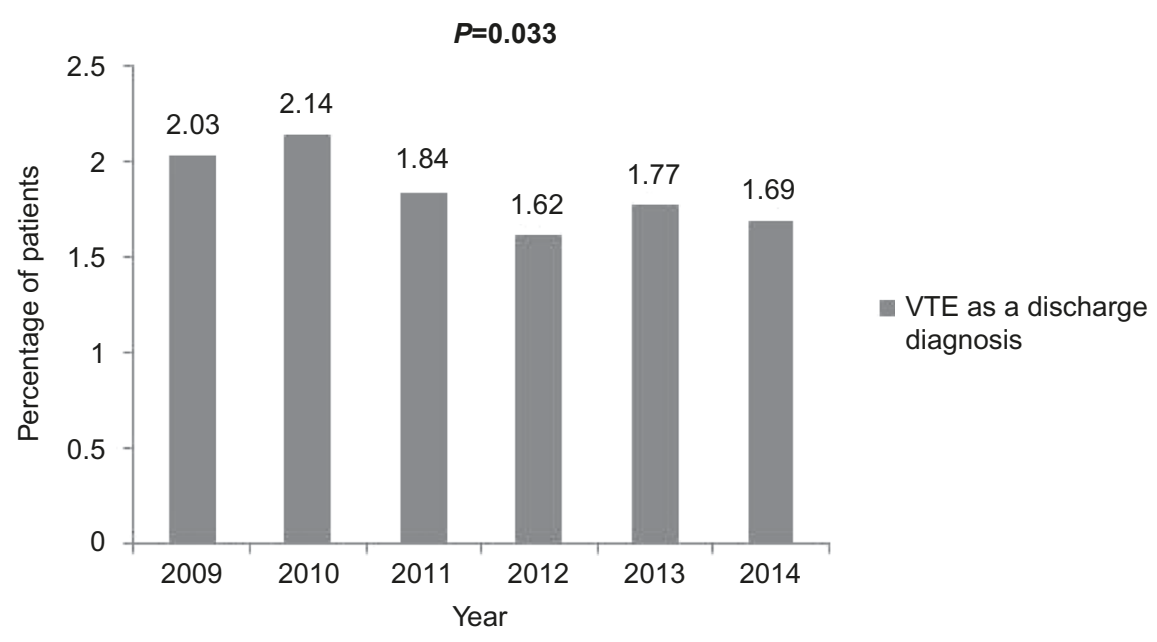

Figure 4 Percent of discharged patients with a primary or secondary diagnosis of VTE from 2009 to 2014.

Abbreviation: VTE, venous thromboembolism.

and during the implementation and post-implementation periods are shown in Figure 4. The prevalence of VTE decreased significantly starting in 2011, ie, during the postimplementation period $(P=0.033)$.

\section{Discussion}

During the planning of the VTE prophylaxis protocol at HSL, a strategy for progressive implementation across the various hospital sectors was selected, which allowed for the correction of flaws and difficulties before introduction in the various units. The participation of the entire multidisciplinary staff, delivery of targeted training, dissemination among the entire clinical staff, and continued analysis of outcomes likely contributed to the high percentage of patients assessed for VTE risk within the first 24 hours after admission at the beginning of the program, as well as to the improvement of the adherence to the prophylaxis recommendations over time. Beginning in 2013, the adherence rates were $>70 \%$, which is higher than those usually reported in the literature and similar to those in developed countries in which VTE prophylaxis is traditionally performed. In the Epidemiologic International Day for the Evaluation of Patients at Risk for VTE in the ENDORSE Global Survey, ${ }^{6}$ the rate of adherence to recommended prophylaxis was $>70 \%$ in only two of 32 countries.

The program was successful from its very beginning, with rates of $86.4 \%$ and $66.8 \%$ for the assessment of VTE risk within the first 24 hours after admission and adherence to prophylaxis recommendations, respectively. Starting in 2011 , ie, the onset of the post-implementation period, the risk assessment rate improved progressively, with a slight, nonsignificant decrease in 2014 (Figures 1 and 2). This decrease was likely due to the introduction of new portable devices for patient data registration into the hospital infor- mation system, which required additional training of the multidisciplinary staff.

The choice of a multiple action strategy, which was supported by the institutional VTE prophylaxis committee and based on complete data recording using computerized systems, in addition to continuous auditing of and feedback to the medical staff and multidisciplinary teams, certainly contributed to the success of the program. Several studies confirm the role of multiple-strategy approaches combined with continued education and constant feedback as a basis for the implementation of health protocols..$^{2,7,9-11}$

From January to December 2009, the prevalence of VTE among hospitalized patients was $2.03 \%$, which likely does not reflect the impact of the morbidity and mortality associated with this diagnosis. A necropsy series revealed the involvement of PE in up to $10 \%$ of in-hospital deaths, ${ }^{1}$ indicating that the incidence of VTE is often underestimated and that preventive actions are crucial for reducing its prevalence. Given the likely increase in the number of hospital admissions of high-risk and older patients, it is necessary to implement a series of preventive actions to reduce VTE rates. ${ }^{12}$ The recent use of decision-making protocols is considered a positive contribution toward improving the quality of care.

Following the implementation of our program, the prevalence of VTE significantly decreased by $\sim 17 \%$. As the data are based on the discharge diagnoses over the investigated period and not on a direct comparison of high-risk patients who were or were not administered prophylaxis, the improvement observed may be due to chance. However, evidence in the literature indicates that the implementation of VTE prophylaxis protocols can improve the rates of adherence to prophylaxis recommendations and reduce the occurrence of clinical VTE events. ${ }^{13,14}$ 


\section{Conclusion}

The implementation of a VTE prophylaxis program targeting adults admitted to a large hospital that employed a multiple-strategy approach achieved high rates of risk assessment within the first 24 hours after admission, improved the adequacy of prophylaxis in high-risk patients, and reduced the rate of VTE events, according to the discharge records.

\section{Acknowledgment}

Karina Ribeiro performed the statistical analysis.

\section{Disclosure}

The authors report no conflicts of interest in this work.

\section{References}

1. Merli GJ. Pulmonary embolism in medical patients: improved diagnosis and the role of low-molecular-weight heparin in prevention and treatment. J Thromb Thrombolysis. 2004;18(2):117-125.

2. Tooher R, Middleton P, Pham C, et al. Systematic review of strategies to improve prophylaxis for venous thromboembolism in hospitals. Ann Surg. 2005;241(3):397-415.

3. Geerts WH, Bergqvist D, Pineo GF, et al. American College of Chest Physicians. Prevention of venous thromboembolism. American College of Chest Physicians Evidence-Based Clinical Practice Guidelines (8th Edition). Chest. 2008;133(6 suppl):381S-453S
4. Pesavento R, Prandoni P. Prevention and treatment of the postthrombotic syndrome and of the chronic thromboembolic pulmonary hypertension. Expert Rev Cardiovasc Ther. 2015;13(2):193-207.

5. Clagett GP, Anderson FA Jr, Heit J, Levine MN, Wheeler HB. Prevention of venous thromboembolism. Chest. 1995;108(4 suppl):312S-334S.

6. Bergmann JF, Cohen AT, Tapson VF, et al. Venous thromboembolism risk and prophylaxis in hospitalised medically ill patients. The ENDORSE Global Survey. Thromb Haemost. 2010;103(4):736-748.

7. Paiva EF, Rocha AT. How to implement a guideline from theory to practice: the example of the venous thromboembolism prophylaxis. Acta Med Port. 2009;22(1):21-32.

8. World Health Organization [webpage on the Internet]. International Statistical Classification of Diseases and Related Health Problems 10th Revision. Available from: http://apps.who.int/classifications/icd10/ browse/2016/en. Accessed August 7, 2010.

9. Fagot J, Flahault A, Fodil M, et al. Impact of expert recommendations on LMWH venous thromboembolism prophylaxis for non-surgery patients on physician prescriptions. Presse Med. 2001;30(5):203-208.

10. Caprini JA, Hyers TM. Compliance with antithrombotic guidelines. Manag Care. 2006;15(9):49-66.

11. Kawamoto K, Houlihan CA, Balas EA, Lobach DF. Improving clinical practice using clinical decision support systems: a systematic review of trials to identify features critical to success. BMJ. 2005;330(7494):765.

12. Roderick P, Ferris G, Wilson K, et al. Towards evidence-based guidelines for the prevention of venous thromboembolism: systematic reviews of mechanical methods, oral anticoagulation, dextran and regional anaesthesia as thromboprophylaxis. Health Technol Assess. 2005;9(49):1-78.

13. Kucher N, Koo S, Quiroz R, et al. Electronic alerts to prevent venous thromboembolism among hospitalized patients. $N$ Engl J Med. 2005;352(10):969-977.

14. Durieux P, Nizard R, Ravaud P, Mounier N, Lepage E. A clinical decision support system for prevention of venous thromboembolism. JAMA. 2000;283(21):2816-2821.
Vascular Health and Risk Management

\section{Publish your work in this journal}

Vascular Health and Risk Management is an international, peerreviewed journal of therapeutics and risk management, focusing on concise rapid reporting of clinical studies on the processes involved in the maintenance of vascular health; the monitoring, prevention and treatment of vascular disease and its sequelae; and the involvement of

\section{Dovepress}

metabolic disorders, particularly diabetes. This journal is indexed on PubMed Central and MedLine. The manuscript management system is completely online and includes a very quick and fair peer-review system, which is all easy to use. Visit http://www.dovepress.com/ testimonials.php to read real quotes from published authors. 